\title{
FINITE ELEMENT SIMULATIONS OF LOGGING-WHILE-DRILLING AND EXTRA-DEEP AZIMUTHAL RESISTIVITY MEASUREMENTS USING NON-FITTING GRIDS
}

\author{
T. CHAUMONT-FRELET ${ }^{1}$, D. PARDO ${ }^{1,2,3}$, AND Á. RODRÍGUEZ-ROZAS ${ }^{1}$ \\ ${ }^{1}$ BASQUE CENTER FOR APPLIED MATHEMATICS \\ ${ }^{2}$ UNIVERSITY OF THE BASQUE COUNTRY \\ ${ }^{3}$ IKERBASQUE
}

\begin{abstract}
We propose a discretization technique using non-fitting grids to simulate magnetic field based resistivity logging measurements. Non-fitting grids are convenient because they are simpler to generate and handle than fitting grids when the geometry is complex. On the other side, fitting grids have been historically preferred because they offer additional accuracy for a fixed problem size in the general case. In this work, we analyse the use of non-fitting grids to simulate the response of logging instruments that are based on magnetic field resistivity measurements using 2.5D Maxwell's equations. We provide various examples demonstrating that, for these applications, if the finite element matrix coefficients are properly integrated, the accuracy loss due to the use of non-fitting grids is negligible compared to the case where fitting grids are employed.
\end{abstract}

\section{INTRODUCTION}

Electromagnetic (EM) logging methods are widely used in oil engineering for their ability to distinguish between oil and water-saturated rocks [16]. Applications include logging while drilling (LWD) [23], deep and extra-deep azimuthal logging [5,30] and crosswell tomography [31]. In these applications, an EM field is generated by a logging instrument placed inside a well. The tool is equipped with EM receivers in order to measure how the generated field is impacted by the resistivity of surrounding rocks. The raw receiver measurements are rarely provided, but instead, they are often post-processed to compute a quantity called "apparent resistivity", which is defined as the resistivity of the infinite homogeneous medium that reproduces the measurements [16]. The apparent resistivity is recorded as the logging instrument is moved along the well, and the resulting curve in which the apparent resistivity is plotted against the tool position is called a "resistivity $\log "$.

In simple geometries where the logging trajectory is almost perpendicular to the discontinuities in resistivity, practitioners can often identify the EM rock properties nearby the well directly from the resistivity log. When the formation geometry is more complex, however, the relation between apparent resistivity and the actual resistivity distribution is not obvious. In addition, borehole effects $[20,22]$, and invasion by drilling mud $[13,28]$ can further affect the measurements.

To properly interpret borehole resistivity measurements in all possible situations, numerical inversion and simulation algorithms are required. Hence, there is a need for accurate, robust and efficient discretization schemes that are able to reproduce resistivity logs. This is especially true in geosteering applications, in which inverted resistivity measurements are used to guide the drilling well trajectory in real time.

Nowadays, real-time fully 3D simulations are out of reach. But they are feasible if additional assumptions are made on the resistivity distribution (see e.g. [26] or [19]). For simplicity of computations, we focus on the case where the resistivity distribution is invariant along one space direction, say $y$. Notice, however, that most results presented here can be trivially applied to fully $3 \mathrm{D}$ scenarios.

Given a 2D conductivity distribution, we apply a Fourier transform along the $y$ direction, and we obtain a set of independent 2D problems, one per Fourier mode $\xi$. The strategy then consists 
in sampling a finite number of Fourier modes $\left\{\xi_{j}\right\}_{j=1}^{N}$ for which the 2D problems are solved. We then recover the electromagnetic fields by performing an approximate inverse Fourier transform using the sampled Fourier modes. As this technique amounts to approximate a 3D problem by a sequence of $2 \mathrm{D}$ problems, it is usually called $2.5 \mathrm{D}[1,2]$.

For each sampled Fourier mode, one Maxwell-type 2D problem has to be numerically approximated. Different families of numerical methods are available, including finite differences $[1,9]$, finite elements [24] and integral equations [2]. In this work, we focus on finite elements discretizations. We partition the computational domain into a finite element mesh over which we define basis functions. We employ tensor product meshes, which greatly simplify the implementation. In addition, efficient linear solvers and domain decomposition techniques can be easily designed for this type of meshes.

Unfortunately, if used directly, tensor product grids are not well adapted to handle complex geometries. Indeed, if the conductivity distribution does not exhibit a Cartesian structure, the conductivity parameter will take different values inside some mesh elements. In particular, inclined layers produce the so-called stair-case approximations [6]. More generally, we shall refer to "nonfitting meshes" to describe those cases in which the physical interfaces of the conductivity model are not aligned with the element edges. Several approaches have been proposed to overcome difficulties due to the use of non-fitting grids, both for finite differences and finite elements. These approaches include homogenization [12] and immersed interface [15] methods.

In this work, we consider finite elements set on non-fitting meshes. The main justification for using non-fitting grids is the analysis performed in [7], where the authors consider 3D Maxwell's equations in a propagation medium that exhibits a constant magnetic permeability, and investigate the approximation properties provided by non-fitting grids for first-order edge finite elements. They show that the use of non-fitting grids (as compared to fitting grids) severely deteriorates the electric field approximation. However, the optimal convergence rate in terms of element size $h$ is preserved for the magnetic field as long as accurate quadrature schemes are employed to integrate the finite element linear system. As a result, the use of non-fitting grids seems appealing to simulate the responses of logging instruments that are based on magnetic field measurements.

The main contribution of the present paper is to assess the performance and accuracy of nonfitting grids to simulate resistivity logs in practical applications. To this end, we consider realistic examples that are representative of LWD and extra-deep azimuthal applications. We propose three different quadrature techniques and discuss their efficiency and accuracy. In addition, the results obtained with non-fitting grids are compared with those resulting from using standard finite element discretizations with fitting meshes.

The paper is organized as follow. Section 2 describes the model problem we solve. The discretization strategy is presented in Section 3. Section 4 is devoted to numerical experiments, and is followed by the conclusions.

\section{Model Problem}

2.1. Logging-While-Drilling instrument. For Logging-While-Drilling applications, we consider a logging device equipped with two transmitters and two receivers. As depicted on Figure 1 , the tool has a symmetric configuration. The receivers are located at a distance $d_{R X}=0.1 \mathrm{~m}$ from the center of symmetry. For the transmitters, the distance is $d_{T X}=0.6 \mathrm{~m}$.

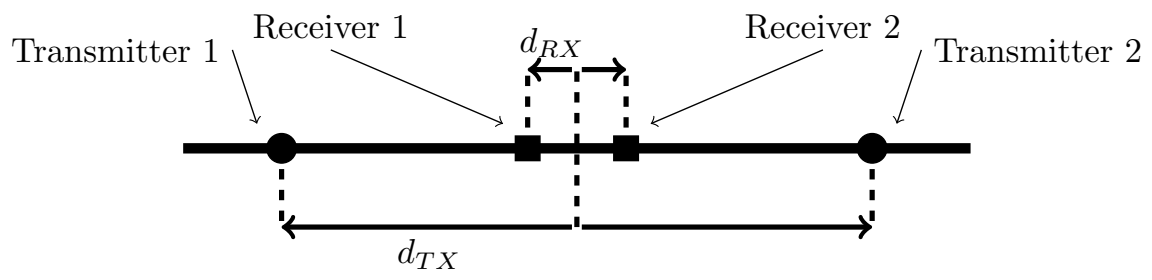

FiguRE 1. Sketch of the tool configuration for LWD 
The transmitters generate alternating currents (AC) with frequency $f=2 \mathrm{MHz}$. The transmitter coils are represented by punctual sources. Similarly, we use the value of the magnetic field at the receivers location to modelize the receivers measurements.

We denote by $\hat{x}, \hat{y}, \hat{z}$ a coordinate system aligned with the logging instrument, $\hat{z}$ being the direction of the tool axis. We denote by $\mathbf{H}_{a b}^{i j}$ (where $a, b=\hat{x}, \hat{y}$ or $\hat{z}$ and $i, j=1$ or 2 ) the magnetic field value recorded by the $j$-th receiver in the $b$ direction when the field is generated by the $i$-th transmitter in the $a$ direction. The attenuation $A_{a b}^{i}$ and the phase difference $P_{a b}^{i}$ measured when the $i-t h$ transmitter is active are then defined via post processing as

$$
A_{a b}^{i}=\operatorname{Re} \log \frac{\mathbf{H}_{a b}^{i 1}}{\mathbf{H}_{a b}^{i 2}}, \quad P_{a b}^{i}=\operatorname{Im} \log \frac{\mathbf{H}_{a b}^{i 1}}{\mathbf{H}_{a b}^{i 2}} .
$$

The final attenuation and phase difference are defined as the average of the values produced by the two transmitters:

$$
A_{a b}=\frac{1}{2}\left(A_{a b}^{1}+A_{a b}^{2}\right), \quad P_{a b}=\frac{1}{2}\left(P_{a b}^{1}+P_{a b}^{2}\right) .
$$

We compute the apparent resistivity by post processing either the attenuation or the phase difference. Since analytical solutions are available in homogeneous media, for a value of $\rho$, one easily estimates the attenuation and phase corresponding to the tool configuration. Hence, the curves $\rho \rightarrow \bar{A}_{a b}(\rho)$ and $\rho \rightarrow \bar{P}_{a b}(\rho)$ in a homogeneous medium can be computed once the tool configuration is known. This is an "off-line" operation that needs to be performed only once per tool configuration.

The apparent resistivities are then obtained as the values $\rho_{a b}^{A}$ and $\rho_{a b}^{P}$ such that $\bar{A}_{a b}\left(\rho_{a b}^{A}\right)=A_{a b}$ or $\bar{P}_{a b}\left(\rho_{a b}^{P}\right)=P_{a b}$, assuming both functions $\bar{A}_{a b}$ and $\bar{P}_{a b}$ are bijective. If any of them is not injective, it leads to a multiple definition of apparent resistivity, while the lack of surjectivity produces the so-called "horns" on the resistivity log.

In applications, all transmitter and receiver directions $(a, b=\hat{x}, \hat{y}, \hat{z})$ are of interest to help identify the resistivity distribution of the formation [10]. In this work, however, for the sake of simplicity we will focus on the coaxial component $a=b=\hat{z}$. Nonetheless, all triaxial components can be naturally handled.

2.2. Deep-azimuthal logging instrument. In deep-azitmuthal logging applications, we consider a logging device equipped with one transmitter and one receiver that are spaced by $25 \mathrm{~m}$, as depicted on Figure 2. The transmitter operates at the frequency $f=2 \mathrm{KHz}$. We use the same modelization techniques than for the LWD tool to represent the transmitter and the receiver.

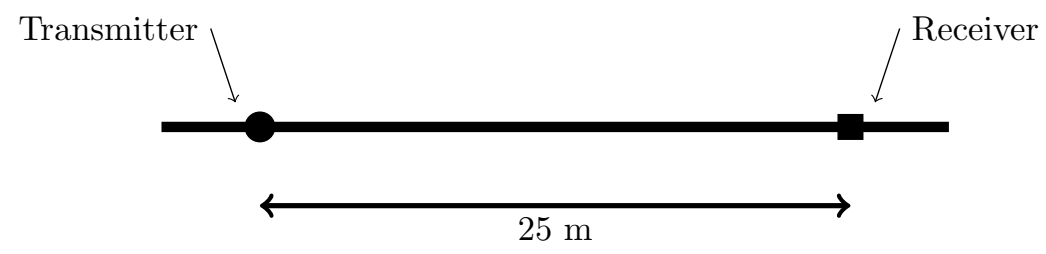

FiguRE 2. Sketch of the tool configuration for deep-azimuthal

We denote by $\hat{x}, \hat{y}, \hat{z}$ a coordinate system aligned with the logging instrument, $\hat{z}$ being the direction of the tool axis. The transmitter is oriented along the tool axis. We consider two configurations for the receiver: one where the $\hat{z}$ component of the magnetic field is recorded $\left(\mathbf{H}_{z z}\right)$, and one where the $\hat{x}$ component is measured $\left(\mathbf{H}_{z x}\right)$.

2.3. The 2.5D Maxwell's system. As the logging instrument emits alternating currents (AC) at frequency $f$, time-harmonic Maxwell's equations are used to modelize the electromagnetic fields. The magnetic field $\mathcal{H}: \mathbb{R}^{3} \rightarrow \mathbb{C}^{3}$ is then characterized as the solution to the reduced wave equation given by

$$
i \omega \mu_{0} \mathcal{H}+\nabla \times(\tilde{\boldsymbol{\rho}} \boldsymbol{\nabla} \times \mathcal{H})=\mathcal{M} \text { in } \mathbb{R}^{3}
$$


where $\mathcal{M}$ is a magnetic dipole representing the transmitter. We consider a transverly isotropic (TI) medium, so that

$$
\tilde{\boldsymbol{\rho}}=\left(\begin{array}{ccc}
\tilde{\rho}_{h} & 0 & 0 \\
0 & \tilde{\rho}_{h} & 0 \\
0 & 0 & \tilde{\rho}_{v}
\end{array}\right)=\left(\begin{array}{ccc}
i \omega \varepsilon_{0}+\rho_{h} & 0 & 0 \\
0 & i \omega \varepsilon_{0}+\rho_{h} & 0 \\
0 & 0 & i \omega \varepsilon_{0}+\rho_{v}
\end{array}\right),
$$

and $\rho_{h}, \rho_{v}: \mathbb{R}_{3} \rightarrow \mathbb{R}_{+}^{*}$ are the horizontal and vertical resistivies, respectively. $\varepsilon_{0}=8.854 \times 10^{-12}$ $\mathrm{Fm}^{-1}$ and $\mu_{0}=4 \pi \times 10^{-7} \mathrm{NA}^{-2}$ are the vacuum permittivity and permeability, $\omega=2 \pi f$ is the angular frequency and $i$ is the imaginary unit $\left(i^{2}=-1\right)$. In addition to (1), the magnetic field $\mathcal{H}$ should decay (exponentially fast) as approaching infinity. To simplify the mathematical analysis, we represent the magnetic dipole by a volumetric source. Here, we select a Gaussian load to represent such magnetic dipole. Thus, $\mathcal{M}=g \mathbf{d}$, where $g$ is a $3 \mathrm{D}$ Gaussian function and $\mathbf{d}$ a unit vector that represents the direction of the transmitter. The actual amplitude of the source is irrelevant, since phase differences and attenuations are insensitive to source amplitude variations.

We assume a two dimensional (2D) resistivity distribution. Hence, we write $\boldsymbol{\rho}(x, y, z)=\boldsymbol{\rho}(x, z)$ where the $y$ direction is arbitrary. We perform a Fourier transform with respect to $y$. Because $\boldsymbol{\rho}$ does not depend on $y$, the resulting equations decouple for each Fourier mode. Thus, for $\xi \in \mathbb{R}$, we introduce

$$
\hat{\mathcal{H}}^{\xi}(x, z)=\int_{-\infty}^{+\infty} \mathcal{H}(x, y, z) e^{i \xi y} d y .
$$

For each $\xi \in \mathbb{R}, \hat{\mathcal{H}}^{\xi}$ is a vector with three components depending on variables $x$ and $z$. We introduce $H_{y}^{\xi}=\hat{\mathcal{H}}_{y}^{\xi}$ and $\mathbf{H}^{\xi}=\left(\hat{\mathcal{H}}_{x}^{\xi}, \hat{\mathcal{H}}_{z}^{\xi}\right)$, so that $H_{y}^{\xi}$ is a $2 \mathrm{D}$ scalar function and $\mathbf{H}^{\xi}$ is a $2 \mathrm{D}$ vector field.

Starting from 3D problem (1), we have that for each fixed $\xi \in \mathbb{R}$, the pair $\left(\mathbf{H}^{\xi}, H_{y}^{\xi}\right)$ is solution to the following $2 \mathrm{D}$ problem:

$$
\left\{\begin{aligned}
\left(i \omega \mu_{0} \mathbf{I}_{2}+\xi^{2} \tilde{\boldsymbol{\rho}}\right) \mathbf{H}^{\xi}+\operatorname{curl}\left(\tilde{\rho}_{h} \operatorname{curl} \mathbf{H}^{\xi}\right)-i \xi \tilde{\boldsymbol{\rho}} \boldsymbol{\nabla} H_{y}^{\xi} & =\mathbf{M}^{\xi} \\
i \omega \mu_{0} H_{y}^{\xi}-\boldsymbol{\nabla} \cdot\left(\tilde{\boldsymbol{\rho}} \nabla H_{y}^{\xi}\right)+i \xi \nabla \cdot\left(\tilde{\boldsymbol{\rho}} \mathbf{H}^{\xi}\right) & =M_{y}^{\xi},
\end{aligned}\right.
$$

where $\mathbf{M}^{\xi}=\left(\mathbf{d}_{x}, \mathbf{d}_{z}\right) \hat{g}^{\xi}$ and $M_{y}=d_{y} \hat{g}^{\xi}$ and $\hat{g}^{\xi}$ stands for the Fourier transform of the Gaussian load $g$. Also, we have kept the notation $\tilde{\boldsymbol{\rho}}$, and introduced the notation $\mathbf{I}_{2}$ to denote the $2 \times 2$ matrices

$$
\tilde{\boldsymbol{\rho}}=\left(\begin{array}{cc}
\tilde{\rho}_{h} & 0 \\
0 & \tilde{\rho}_{v}
\end{array}\right), \quad \mathbf{I}_{2}=\left(\begin{array}{cc}
1 & 0 \\
0 & 1
\end{array}\right)
$$

In addition to (3), a decaying condition is satisfied at infinity. To mimic that condition, we artificially bound the domain of computation to numerically approximate the problem with finite elements. The artificial boundary only introduces a small modelization error when placed sufficiently far from the magnetic dipole. We thus introduce a domain $\Omega$ which includes the magnetic dipole. We select the boundary $\partial \Omega$ such that it is sufficiently far from the source. Then, we prescribe the following boundary conditions on $\partial \Omega$

$$
\mathbf{H}^{\xi} \cdot \mathbf{n}=0, \quad \nabla H_{y}^{\xi} \cdot \mathbf{n}=0,
$$

where $\mathbf{n}$ is the unit vector normal to $\partial \Omega$ pointing outward $\Omega$.

Taking into account boundary conditions (4), we reformulate (3) into the following variational problem: Find $\left(\mathbf{H}^{\xi}, H_{y}^{\xi}\right) \in H(\operatorname{curl}, \Omega) \times H^{1}(\Omega)$ such that

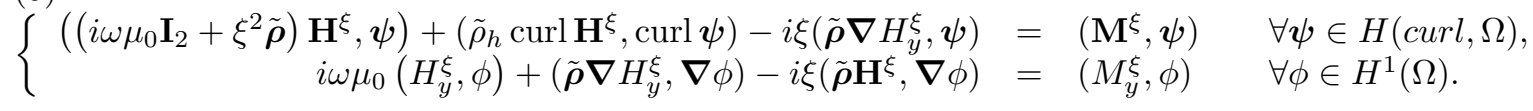

For general geometries, one can not solve (5) analytically. It is thus required to numerically approximate its solution. In this work, we use a finite element method described in Section 3.

In addition, we do not approximate the solution to problem (5) for all Fourier modes $\xi \in \mathbb{R}$. Instead, we restrict to a finite number of selected Fourier modes and recover the magnetic field 
using an approximate inverse Fourier transform:

$$
\mathcal{H}(x, y, z) \simeq \sum_{j=1}^{N_{f}} w_{j} \hat{\mathbf{H}}^{\xi_{j}}(x, z) e^{-\xi_{j} y},
$$

where $\left\{\xi_{j}\right\}_{j=1}^{N_{f}}$ are $N_{f}$ selected Fourier modes, and $\left\{w_{j}\right\}_{j=1}^{N_{f}}$ are associated weights.

\section{The finite Element method}

3.1. Finite element spaces. We use a finite element method to approximate variational formulation (5) of 2.5D Maxwell's equations. The tangential and normal components of the approximated field respectively belong to $H($ curl,$\Omega)$ and $H^{1}(\Omega)$. As a result, we employ Nédélec edge elements [21] to approximate the tangential component, and Lagrange nodal elements [8] to approximate the normal component (in the $y$ direction).

For the sake of simplicity, we consider tensor product grids composed of squared elements. The presented method can be naturally extended to curved and/or triangular elements, as discussed in Section 3.2.

For a selected mesh $\mathcal{T}_{h}$, the Lagrange nodal element space for $H_{y}^{\xi}$ is defined as

$$
S^{h, p}=\left\{v_{h, p} \in H^{1}(\Omega) \mid v_{h, p} \in Q_{p p}(K) \forall K \in \mathcal{T}_{h}\right\} .
$$

where $p \geq 1$ is the polynomial degree. For the tangential component $\mathbf{H}^{\xi}$, we introduce the Nedelec edge element space

$$
\mathbf{V}^{h, p}=\left\{\mathbf{v}_{h, p} \in H(\operatorname{curl}, \Omega) \mid \mathbf{v}_{h, p} \in Q_{p(p-1)}(K) \times Q_{(p-1) p}(K) \forall K \in \mathcal{T}_{h}\right\} .
$$

In the above definitions, $Q_{r q}$ stands for the space of scalar polynomials of degree at most $r$ in the $x$ variable and at most $q$ in the $z$ variable.

The discrete version of problem (5) is obtained by replacing the continuous functional spaces $H(\operatorname{curl}, \Omega)$ and $H^{1}(\Omega)$ by their discrete counterparts $\mathbf{V}^{h, p}$ and $S^{h, p}$.

3.2. Assembly of the linear system and integration. We employ possibly non-fitting meshes. Authors of [7] demonstrate that non-fitting meshes are able to produce accurate approximations of the magnetic field. Specifically, they show that for first-order Nedelec's elements, the magnetic field approximation converges linearly in $L^{2}$-norm, both for fitting and non-fitting meshes. This legitimate further investigations around the use of non-fitting grids to simulate the response of logging instruments that are based on magnetic measurements.

The analysis presented in [7] assumes that the coefficients of the finite element matrix are integrated exactly. For fitting meshes, performing the integration of the linear system coefficients is simple. Indeed, since the resistivity parameter is constant (or at least smooth) inside each cell, it is sufficient to select a quadrature scheme that integrates polynomials of sufficiently high degree over a square. For non-fitting meshes, however, the resistivity parameter can jump inside mesh cells, and the behaviour of traditional quadrature schemes in terms of accuracy is unclear.

In order to simplify the discussion, we focus on one particular term. In each element $K$, we need to integrate the contributions of basis functions $\phi_{i}, \phi_{j} \in S^{h, p}$ that involve the quantities

$$
\int_{K} \tilde{\rho}_{h} \operatorname{curl} \phi_{j} \operatorname{curl} \phi_{i}
$$

For a fitting mesh, $\left.\tilde{\rho}_{h}\right|_{K} \in \mathbb{R}$ is constant inside $K$. As a result, we can extract $\tilde{\rho}_{h}$ from integral (7), and then, it simply remains to integrate a polynomial quantity over a square. This can be done analytically. Furthermore, optimized strategies involving a "master element" and affine mappings make it possible to compute $(7)$ fast (see $[8,17]$ for instance).

On the other hand, when the mesh is non-fitting, computing (7) is more challenging, since $\tilde{\boldsymbol{\rho}}$ can take several values inside $K$. A popular approach to compute the integral is then to introduce a homogenized (or, in some sense, averaged) parameter $\tilde{\boldsymbol{\rho}}_{K}^{\star}$ that is constant over $K$. However, it is challenging to select a particular averaging strategy, as several of them are available $[12,14,29]$. In addition, most averaging strategies are rigorously established under some restrictive assumption of the parameter $\tilde{\boldsymbol{\rho}}$. Examples include one dimensional variations [3,18] or small-scale periodicity [14]. 


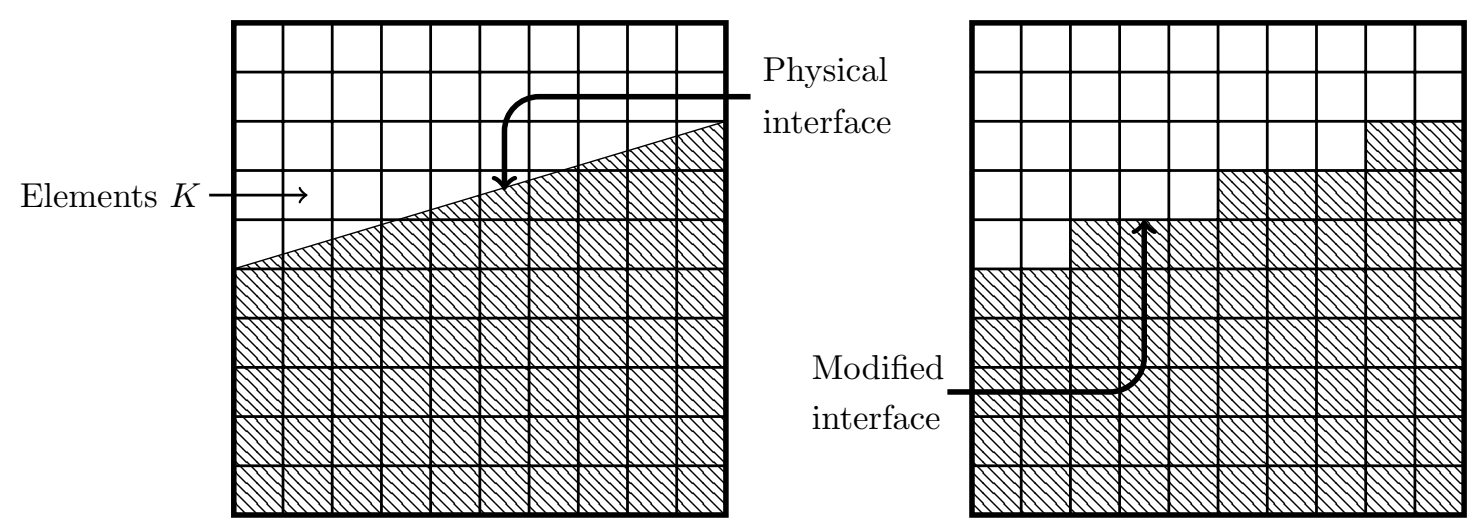

FiguRE 3. Transformation of a physical interface by the constant parameter integration technique

While the resulting averaging formula can be used in more general media, the accuracy of the resulting homogenized approximation is not guaranteed.

In this work, we employ a different strategy to approximate integral (7) that is purely based on quadrature techniques. We do not modify the parameter $\tilde{\boldsymbol{\rho}}$, but instead, we use a sufficiently accurate quadrature scheme to integrate (7) directly. In the following, we investigate three different strategies:

(a) "Constant parameter integration": we approximate $\tilde{\rho}_{h}$ by a constant value over each cell. Specifically, we employ the value of $\tilde{\rho}_{h}$ at the center $\mathbf{x}_{K}$ of the element $K$. Thus, constant parameter integration reduces to

$$
\int_{K} \tilde{\rho}_{h} \operatorname{curl} \phi_{j} \operatorname{curl} \phi_{i} \simeq \tilde{\rho}_{h}\left(\mathbf{x}_{K}\right) \int_{K} \operatorname{curl} \phi_{j} \operatorname{curl} \phi_{i},
$$

This strategy is simple and can be rapidly evaluated using affine mappings from a "master element" (see [17]). As depicted in Figure 3, this method amounts to introduce a so-called "staircase" approximation.

(b) "Gaussian integration": we use the formula

$$
\int_{K} \tilde{\rho}_{h} \operatorname{curl} \phi_{j} \operatorname{curl} \phi_{i} \simeq \sum_{k=1}^{N} w_{k} \tilde{\rho}_{h}\left(\mathbf{x}_{k}\right) \operatorname{curl} \phi_{j}\left(\mathbf{x}_{k}\right) \operatorname{curl} \phi_{i}\left(\mathbf{x}_{k}\right),
$$

where $\left\{\mathbf{x}_{k}\right\}_{k=1}^{N}$ and $\left\{w_{k}\right\}_{k=1}^{N}$ are Gaussian quadrature points and weights that exactly integrate polynomials of $Q_{2 p+1,2 p+1}(K)$. Actually, (9) is just the widely used Gaussian quadrature technique. When the parameter $\tilde{\rho}_{h}$ is smooth, it is known that the quadrature error is small and below the order of approximation. Thus, the quadrature error does not deteriorate the accuracy of the finite element solution. However, for non-fitting grids, the error caused by the quadrature rule might be more important, as the parameter $\tilde{\rho}_{h}$ we consider features discontinuities. Gaussian integration (9) has the advantage to work for non-affine mappings, however, it is more costly than (8) in the case of affine mappings. In addition, there is no clear geometrical interpretation of the approximation committed on the resistivity as in the case of constant parameter integration.

(c) "Subelements integration technique": we introduce a set of squared subelements $\left\{C_{s}\right\}_{s=1}^{S}$ for each element $K$. Then, the integration is performed as

$$
\int_{K} \tilde{\rho}_{h} \operatorname{curl} \phi_{j} \operatorname{curl} \phi_{i} \simeq \sum_{s=1}^{S} \tilde{\rho}_{h}\left(\mathbf{x}_{C}^{s}\right) \int_{C_{s}} \operatorname{curl} \phi_{j} \operatorname{curl} \phi_{i}
$$

where $\mathbf{x}_{C}^{s}$ is the center of $C_{s}$. The last integral in the right-hand-side is easily computed, since the subelements $C_{s}$ are squares. In addition, if affine mappings are used, an efficient strategy involving "master subelements" has been designed in [4] to compute (10). We 


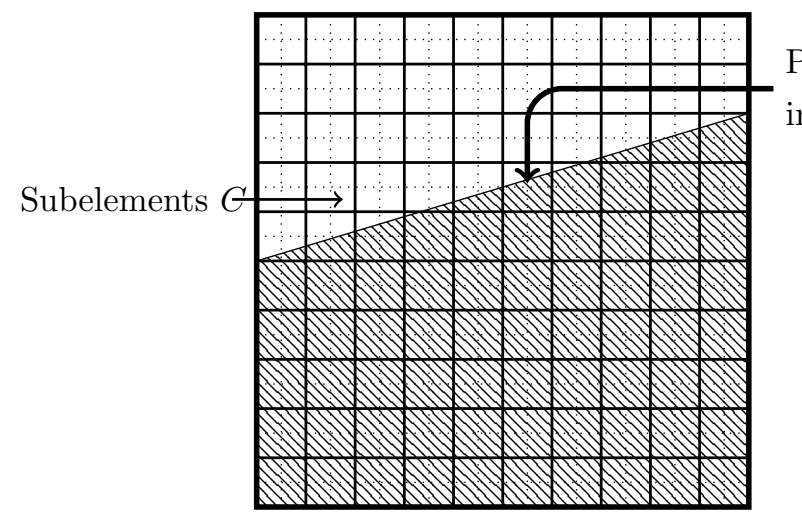

Physical
interface

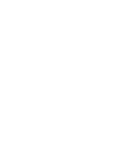

Modified

interface

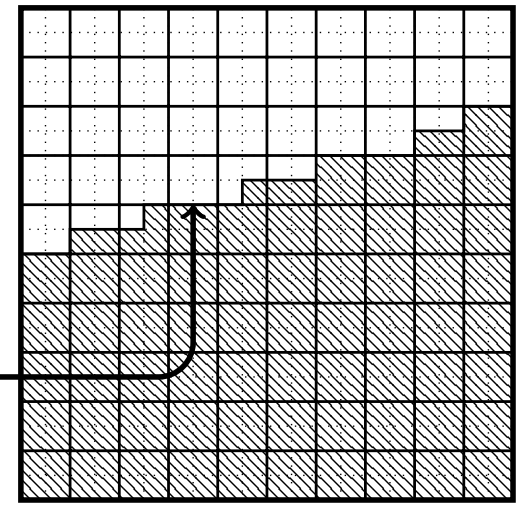

Figure 4. Transformation of a physical interface by the subelements integration technique

observe that if $s=1$, then $C_{1}=K$ and we recover the constant parameter integration technique. (10) is thus a generalization of (8), where the parameter $S$ can be tuned to achieve the desired integration accuracy. Figure 4 shows that this strategy provides a refined staircase approximation of the resistivity distribution.

The presented quadrature techniques can actually be employed on more general meshes. As presented in [4], the Constant parameter, and Subelement integration techniques naturally extend to triangular (or tetrahedral) meshes, as long as the elements are straight (e.g. defined with affine mappings). On the other hand, general curved quadrangular elements can be used together with the Gaussian integration technique.

3.3. Comparison with 3D approaches. In this section, we discuss the computational cost of the proposed 2.5D approach compared to a standard 3D FEM discretization. We assume here that a direct solver is used to factorize the finite element linear system(s), and that the factorization(s) represent(s) most of the computational cost.

Each factorization requires $\mathcal{O}\left(\left(N_{d o f}^{(d-1) / d}\right)^{3}+N_{d o f} p^{2 d}\right)$ floating point operations (FLOPs), where $d$ is the number of spatial dimensions, $p$ is the polynomial degree, and $N_{d o f}$ is the number of degrees of freedom in the linear system $[11,25]$.

If we assume that each spatial dimension is subdivided into $n$ elements, then $N_{\text {dof }}=\mathcal{O}\left((n p)^{d}\right)$. As a result, for a 3D FEM problem, the factorization requires $\mathcal{O}\left(n^{6} p^{6}\right)$ FLOPs, while the cost for a 2D FEM problem is $\mathcal{O}\left(n^{3} p^{3}+n^{2} p^{6}\right)$ FLOPs.

Since we must solve one $2 \mathrm{D}$ problem for each Fourier mode, we obtain that the cost of all factorizations for the 2.5D approach is $\mathcal{O}\left(N_{f} n^{3} p^{3}+N_{f} n^{2} p^{6}\right)$, where $N_{f}$ is the number of Fourier modes.

Since the solution is expected to converge exponentially fast as a function of the number of Fourier modes, we have $N_{f} \ll n$ in applications (for instance, in our LWD experiments $n \simeq 100$ and $N_{f} \simeq 30$ ), the $2.5 \mathrm{D}$ approach employs less than $\mathcal{O}\left(n^{4} p^{3}+p^{6} n^{3}\right)$ FLOPs. As a result, the 2.5D approach requires at least $n^{2}$ less FLOPs than the 3D approach. In addition, the computations associated with each Fourier mode are independent, so that one can easily implement fully parallel algorithms for the $2.5 \mathrm{D}$ approach.

\section{NUMERICAL EXPERIMENTS FOR LOGGING-WHILE-DRILLING}

4.1. Grid design. Our aim is to compare the accuracy of fitting grids versus non-fitting grids, and, for the case of non-fitting grids, to evaluate the errors committed by the use of non-exact integration techniques. To this end, we will use the coarsest possible grids that provide physically meaningful solutions. Indeed, for coarser grids, the non-fitting effect is more important. Thus, coarse grids represent the "worst case scenario". 
In order to use coarse grids, we employ high order finite elements. In the following, we select the value $p=4$. In addition, we use a different grid for each tool position. Such grids are more refined nearby the tool center than close to the domain boundary.

For a particular experiment, we consider the highest resistivity value $\rho_{\max }$ present in the propagation medium. We adapt the grid so that it is able to correctly reproduce the resistivity $\log$ in a homogeneous medium of resistivity $\rho_{\max }$ with an accuracy of $1 \%$.

The grid is also adapted to each Fourier mode. We have empirically observed that using grids twice larger (both the domain and elements sizes are doubled) for the lowest Fourier mode than for the largest one lead to satisfactory results. Therefore, assuming that the Fourier modes are sampled in the interval $\left[0, \xi_{\max }\right]$, we first fix a grid for the Fourier mode zero. Then, for a higher Fourier mode $\xi$, we apply the scaling

$$
\mathbf{x}_{0} \rightarrow \mathbf{x}_{\xi}=\left(1-\frac{1}{2} \frac{\xi}{\xi_{\max }}\right) \mathbf{x}_{0}
$$

to the grid.

Finally, the original grid for the Fourier mode zero is obtained following a manual "trial and error" approach. In our validation experiments, the maximum resistivity is $10 \Omega \cdot \mathrm{m}$. In this case, we end up with the grid size:

$$
h(\mathbf{x})= \begin{cases}0.08 \mathrm{~m} & \text { if }|\mathbf{x}|<0.5 \mathrm{~m} \\ 0.1 \mathrm{~m} & \text { if } 0.5 \mathrm{~m}<|\mathbf{x}|<0.8 \mathrm{~m} \\ 0.11 \mathrm{~m} & \text { if } 0.8 \mathrm{~m}<|\mathbf{x}|<0.9 \mathrm{~m} \\ 0.8 \mathrm{~m} & \text { if } 0.9 \mathrm{~m}<|\mathbf{x}|<2 \mathrm{~m} .\end{cases}
$$

In addition, we truncate the $y$ direction with the length $2 \pi$, and we select the Fourier modes $\xi_{j}=0, \ldots, 25$.

In the more realistic experiment we consider, the maximum resistivity is $100 \Omega \cdot \mathrm{m}$, and the corresponding grid size is:

$$
h(\mathbf{x})= \begin{cases}0.05 \mathrm{~m} & \text { if }|\mathbf{x}|<0.5 \mathrm{~m} \\ 0.075 \mathrm{~m} & \text { if } 0.5 \mathrm{~m}<|\mathbf{x}|<0.75 \mathrm{~m} \\ 0.1 \mathrm{~m} & \text { if } 0.75 \mathrm{~m}<|\mathbf{x}|<1 \mathrm{~m} \\ 2.0 \mathrm{~m} & \text { if } 1 \mathrm{~m}<|\mathbf{x}|<4 \mathrm{~m}\end{cases}
$$

We also select a larger length for $y$-direction in this experiment: $4 \pi$. As a result, we employ the Fourier modes

$$
\xi_{j}=\frac{j}{2}, \quad j=0, \ldots, 30 .
$$

4.2. Validation experiments. We first consider isotropic layers with parallel interfaces. The logging tool follows a straight trajectory and crosses the layers interfaces with an angle $\theta$.

In order to evaluate the accuracy loss due to the use of non-fitting grids, we propose two different scenarios, which are physically equivalent, but that are handled differently by numerical approximations. Figure 5 depicts these cases.

In the first scenario, the layers interfaces are horizontal, and the tool follows a straight path in the direction of the unit vector $(\cos (\theta), \sin (\theta))$. In this case, since the interfaces are horizontal, they are easily fitted by a Cartesian grid.

In the second case, the tool position is horizontal, but the interfaces follow the direction $(\cos (-\theta), \sin (-\theta))$. Since the relative angle between the tool trajectory and the physical interfaces is the same as before, the physical situation is identical. However, the numerical approximation will be different.

4.2.1. Two half-spaces. We consider a propagation medium composed of two half-spaces. In the upper half-space, the resistivity value is $1 \Omega \cdot \mathrm{m}$, while the value of $10 \Omega \cdot \mathrm{m}$ is prescribed in the lower layer. The angle between the tool trajectory and the layer interface is $\theta=10^{\circ}$. Figure 6 shows the resistivity logs corresponding to fitting meshes and non-fitting meshes using different quadrature techniques. 

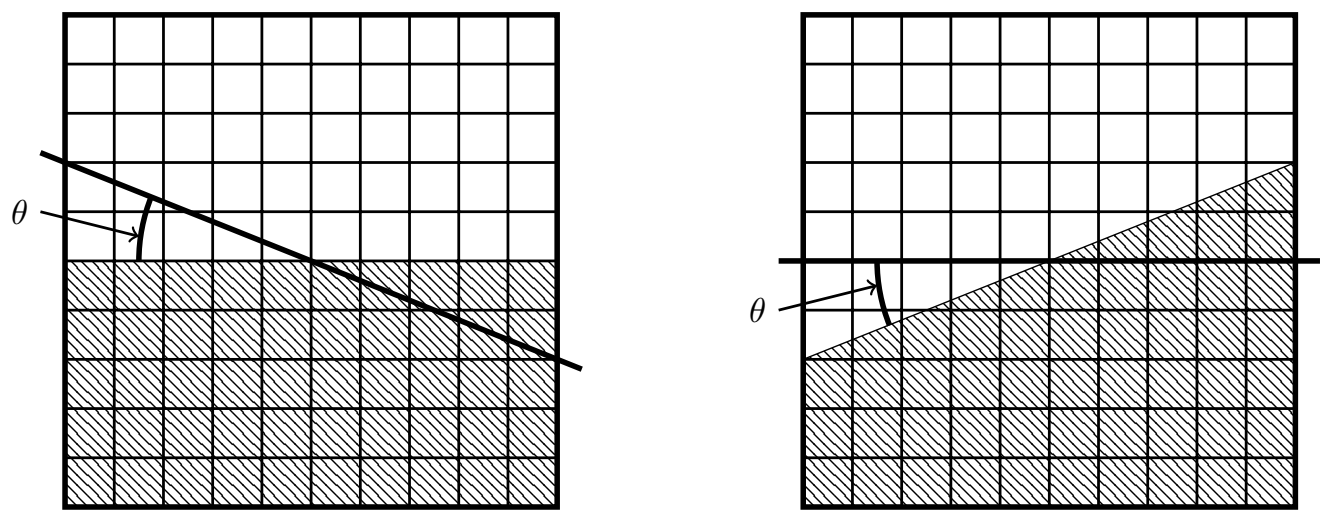

FiguRE 5. Fitting (left) and non-fitting (right) grids for the validation experiments

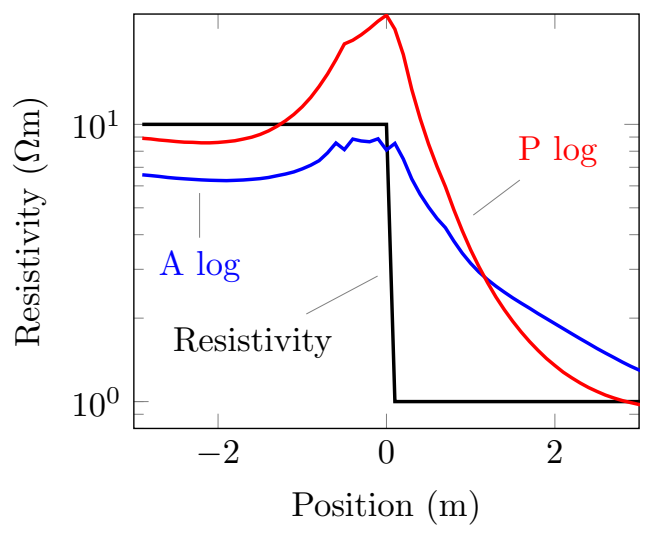

(a) Fitting mesh

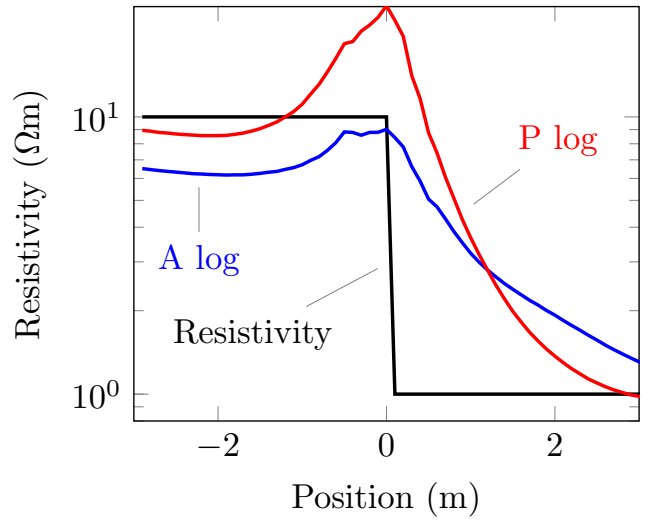

(c) Gaussian integration

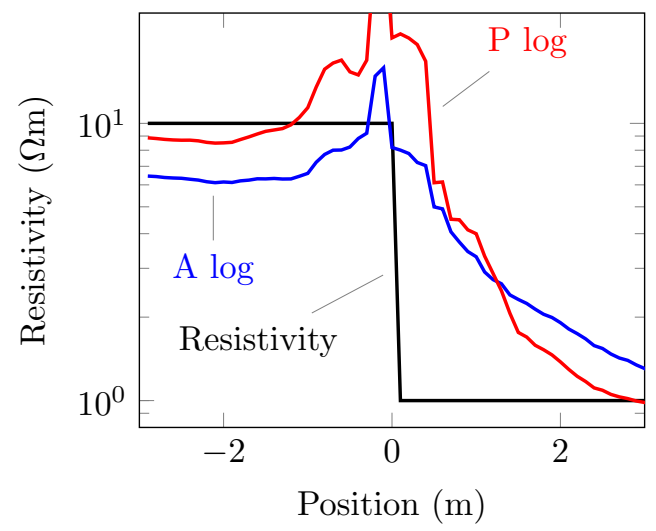

(b) Constant integration

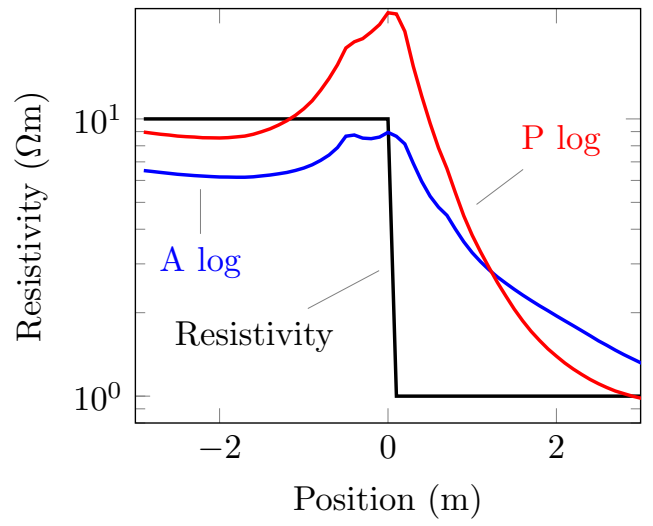

(d) Subelement integration

FiguRE 6. Resistivity log simulations for two half spaces

We employ a finer "overkilling" fitting grid to estimate the errors committed on the A-log and P-log. Figures 7 and 8 describe those errors as a function of the tool position.

The "constant parameter" quadrature technique leads to inaccurate logs when the tool is close to the interface. We further see that for the cases of Gaussian or Subelements integration techniques, non-fitting meshes provide a level of accuracy comparable to that achieved by fitting meshes. 

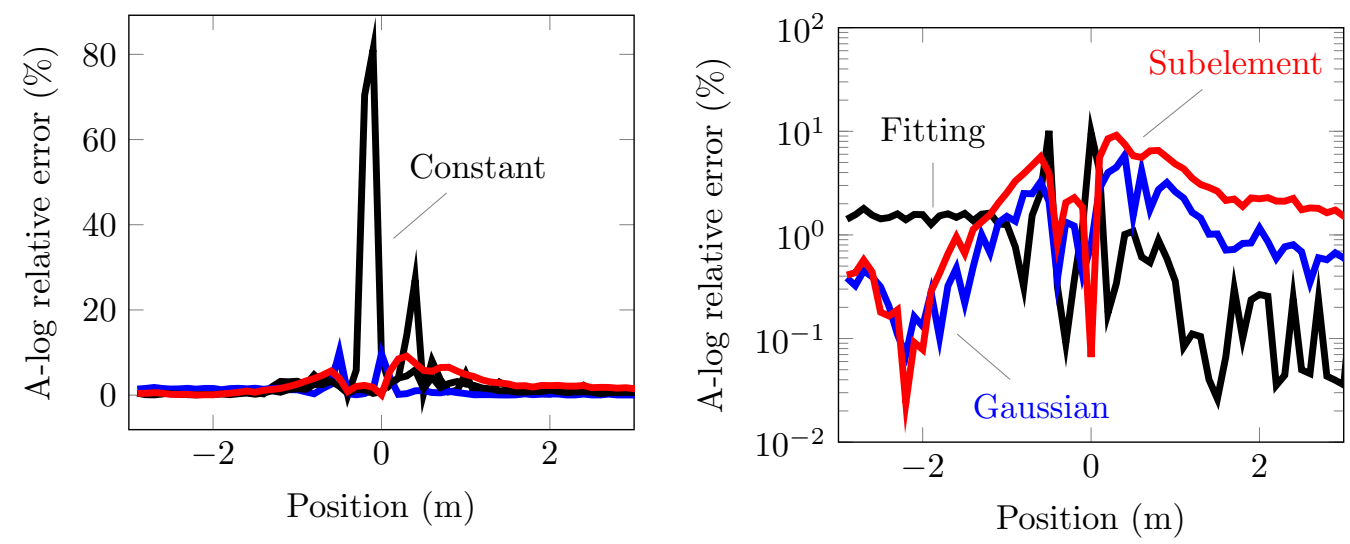

FiguRE 7. Relative errors for the A-log. Formation composed of two half spaces. Left panel: Figure showing the large error produced by the constant parameter integration. Right panel: Figure comparing the errors produced by the other integration techniques vs. the one committed by the fitting mesh discretization.
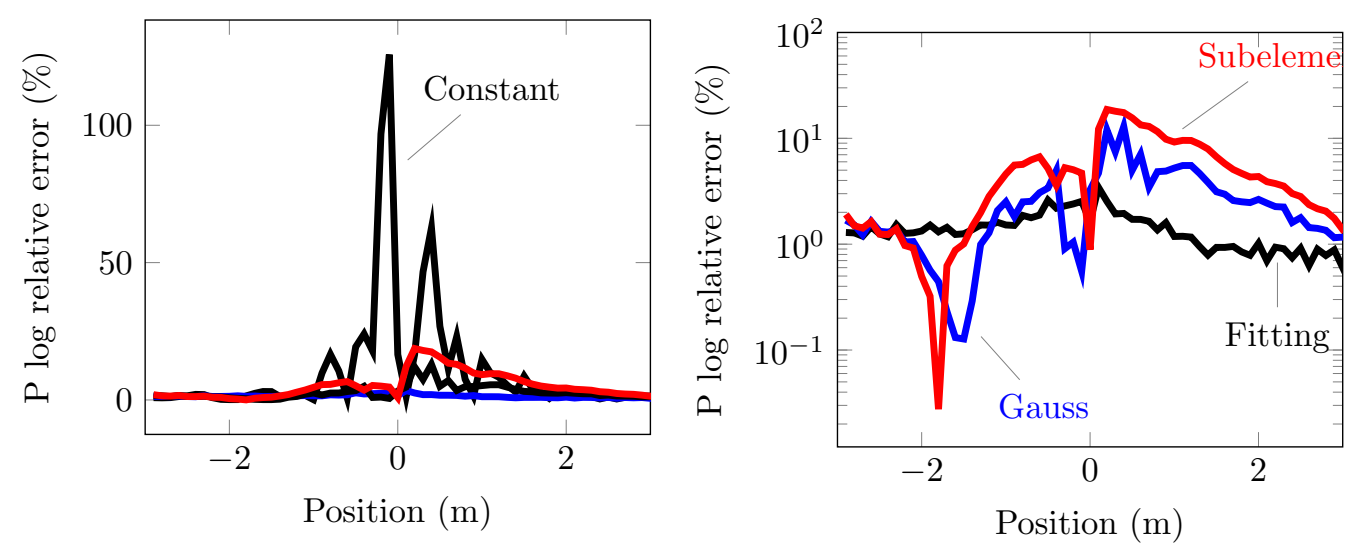

Figure 8. Relative errors for the P-log. Formation composed of two half-spaces. Left panel: Figure showing the large error produced by the constant parameter integration. Right panel: Figure comparing the errors produced by the other integration techniqes vs. the one committed by the fitting mesh discretization.

4.2.2. A thin layer. We now consider a $5 \mathrm{~cm}$ thick layer of resistivity $10 \Omega \cdot \mathrm{m}$. The layer is embedded into a constant $1 \Omega \cdot \mathrm{m}$ resistivity background. The angle between the tool trajectory and the layer interfaces is $\theta=10^{\circ}$. Figure 9 presents the resistivity logs obtained using fitting and non-fitting meshes.

Figure 10 presents the error committed on the resistivity logs (compared to a reference solution computed on a finer mesh). As in the case of the two half-spaces, we see that constant parameter integration lead to an important accuracy loss. On the other hand, fitting and non-fitting meshes provide comparable accuracy when using Gaussian or Subelement quadrature techniques.

4.3. A realistic synthetic example. We now consider the geophysical medium depicted on Figure 11. The medium contains a $5 \mathrm{~m}$ deep reservoir that is partially saturated with oil and water. In addition, the medium features two geophysical faults that are separated by $30 \mathrm{~m}$. All materials are modelized as isotropic. The tool follows a trajectory of $90 \mathrm{~m}$ that is representative of applications in which one tries to keep the geosteering tool inside the oil saturated part of the reservoir. 


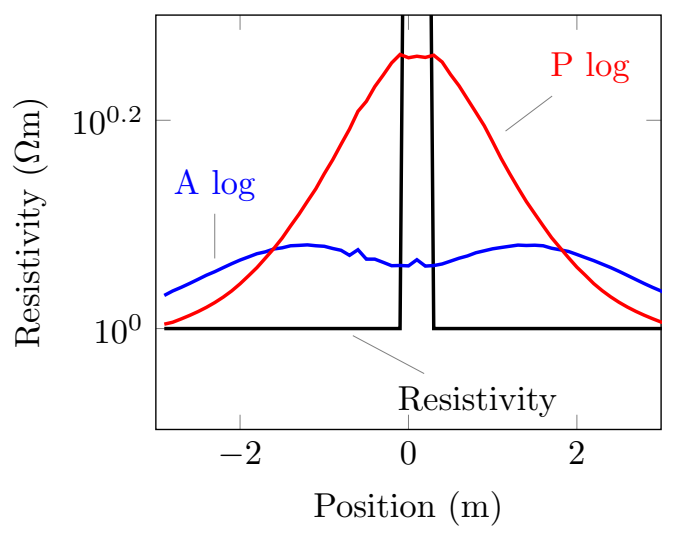

(a) Fitting mesh

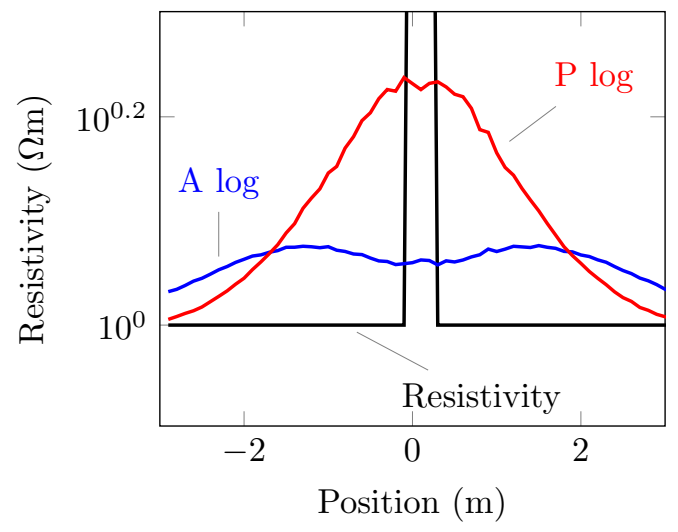

(c) Gaussian integration

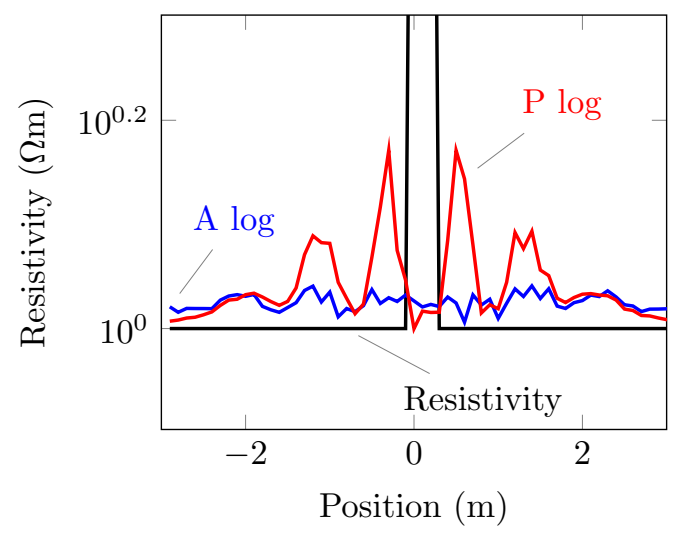

(b) Constant integration

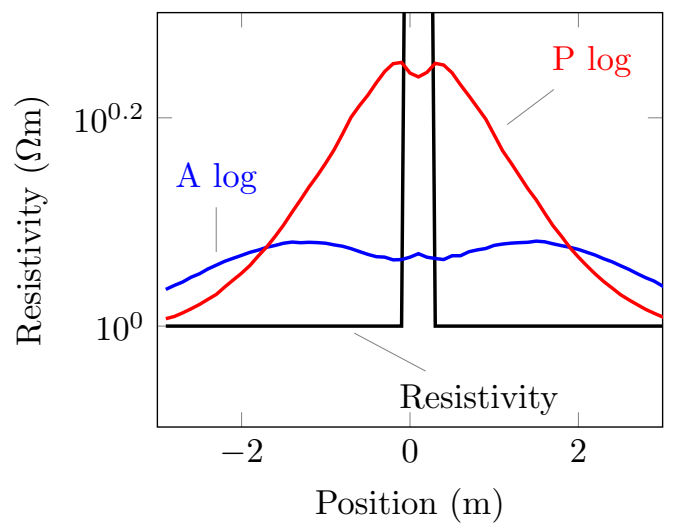

(d) Subelement integration

FiguRE 9. Resistivity log simulations for a thin layer
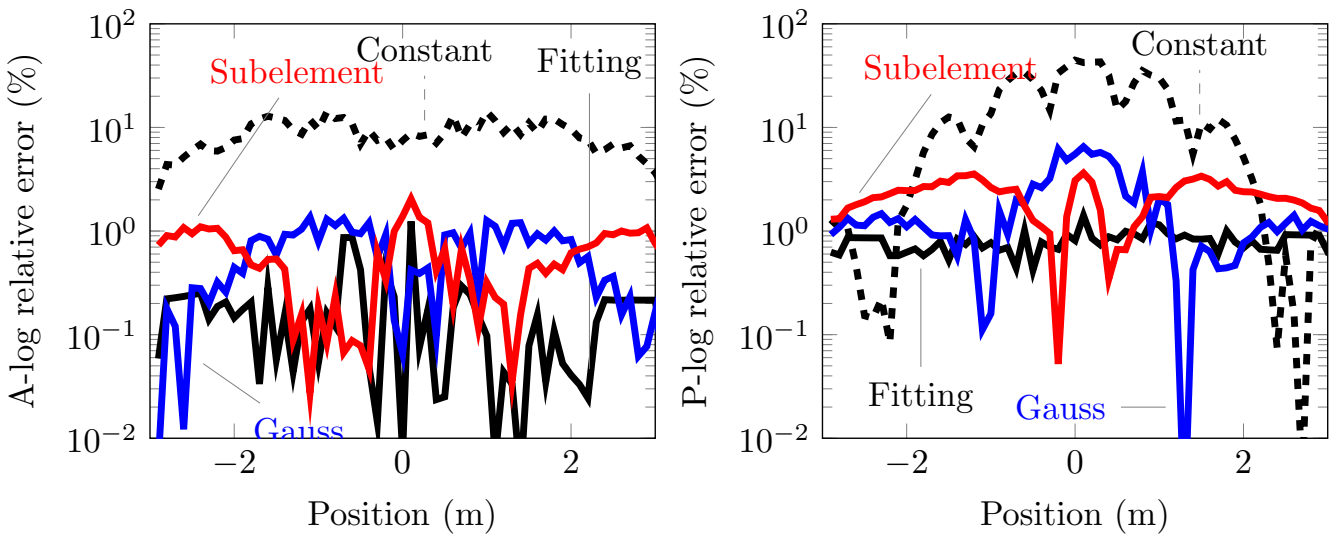

Figure 10. Apparent resistivity errors for the thin layer test case

Figures 12 and 13 describe the simulated A- and P-logs, respectively. We observe that all quadrature methods produce similar logs. Nevertheless, a zoom on a particular area shows that those obtained with the constant parameter integration technique are more "noisy". 


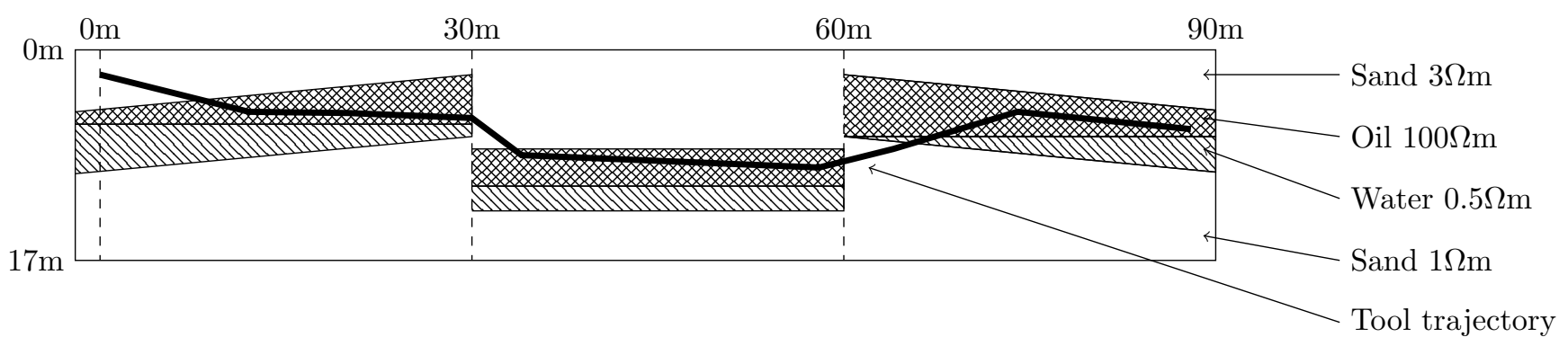

FIGURE 11. Synthetic example comprised of a formation and a geosteering well trajectory

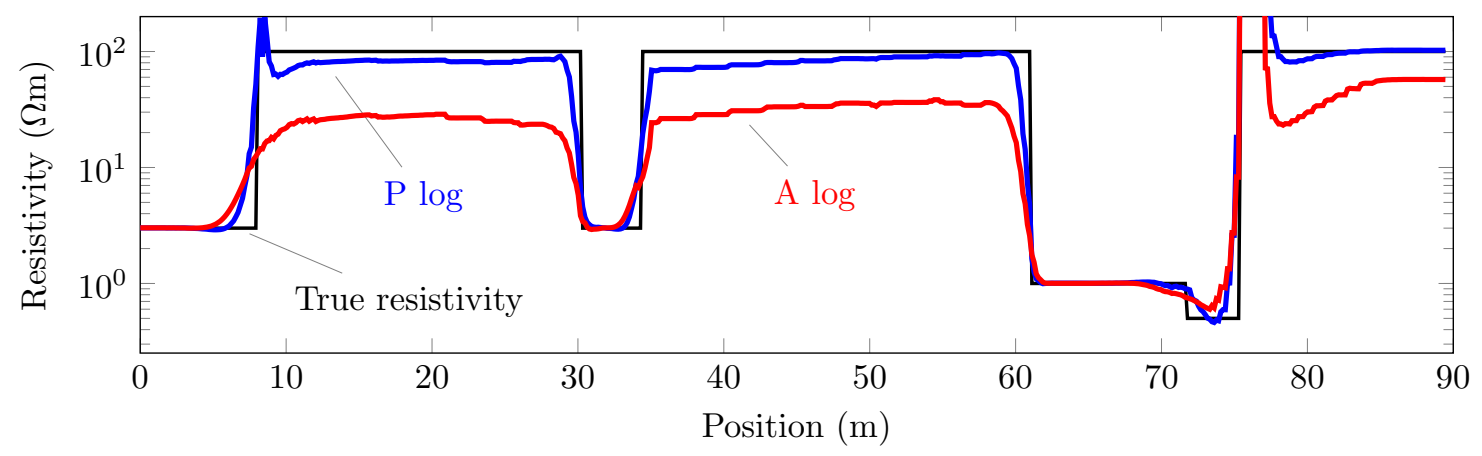

(a) Constant parameter integration

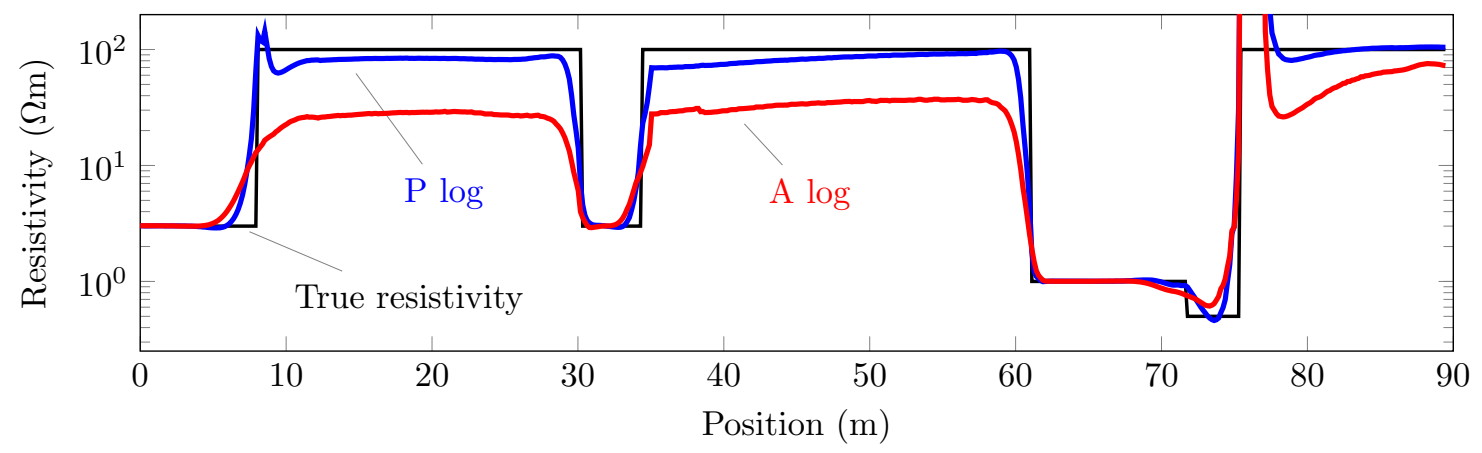

(b) Gaussian integration

Subelement integration

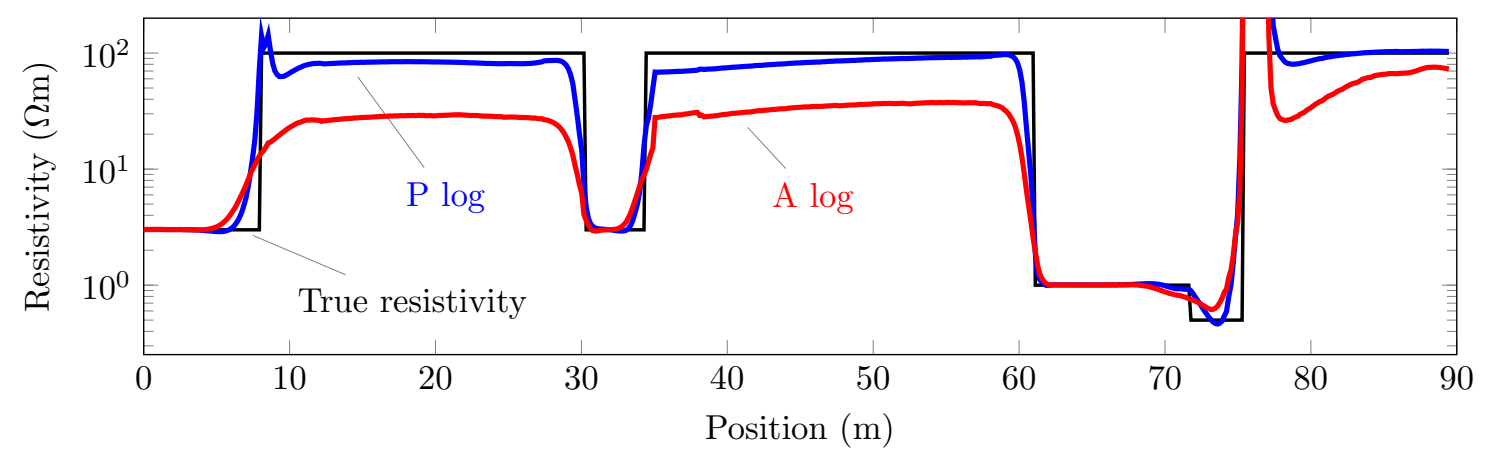

FiguRE 12. Simulated logs (complete trajectory) 


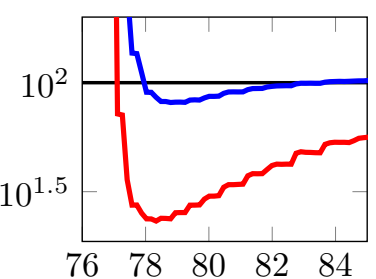

(a) Constant parameter integration

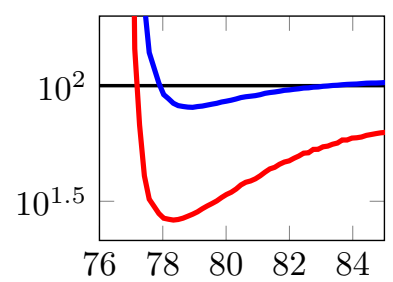

(b) Gaussian integration

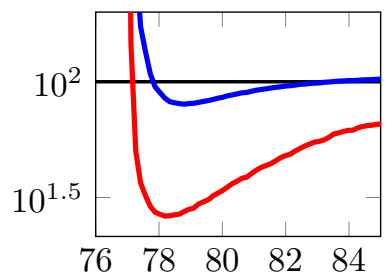

(c) Subelement integration

FiguRE 13. Simulated logs (zoom on a particular area)

\section{NumERICAL EXPERIMENTS FOR DEEP-AZIMUTHAL MEASUREMENTS}

5.1. Grid design. In this experiment, we select $p=2$. We use grids that are refined around both the transmitter and receiver. Specifically, if we denote by

$$
D(\mathbf{x})=\min \left(\left|\mathbf{x}-\mathbf{x}_{R X}\right|,\left|\mathbf{x}-\mathbf{x}_{T X}\right|\right),
$$

the distance between the point $\mathbf{x}$ and the receiver and transmitter, we design the grid so that the local element size satisfies

$$
h(\mathbf{x})= \begin{cases}1 \mathrm{~m} & \text { if } D(x)<5 \mathrm{~m} \\ 2 \mathrm{~m} & \text { if } 5 \mathrm{~m}<D(x)<10 \mathrm{~m} \\ 10 \mathrm{~m} & \text { if } 10 \mathrm{~m}<D(x)<50 \mathrm{~m} .\end{cases}
$$

We select the length $32 \pi \simeq 100 \mathrm{~m}$ in the $y$-direction and the Fourier modes

$$
\xi_{j}=\frac{j}{16}, \quad j=0, \ldots, 60 .
$$

5.2. Validation example. We consider a single experiment that consists of two half-spaces. The top layer is anisotropic, with $\rho_{h}=40 \Omega \cdot \mathrm{m}$ and $\rho_{v}=200 \Omega \cdot \mathrm{m}$. The bottom layer consists of an isotropic material, and we have $\rho_{h}=\rho_{v}=2 \Omega \cdot \mathrm{m}$. The tool follows a straight path, and crosses the interface with an angle $\theta=45^{\circ}$.

Figures 14 and 16 represent the simulated values of $\mathbf{H}_{z z}$ and $\mathbf{H}_{z x}$, respectively. The absolute errors (compared to an "overkill" solution computed with a very fine fitting grid) are displayed on Figures 15 and 17 . We observe that non-fitting grids provide sufficiently accurate results (with errors below the expected noise level), regardless of the integration method.

Due to the large depth-of-investigation of the considered logging instrument, in this example we realize that even the "constant integration" technique leads to highly accurate results in terms of the required engineering precision. We emphasize that in other deep-azimuthal examples, the use of a more precise technique (either Gaussian or sub-integration) may be necessary. Indeed, it is possible to consider a deep-azimuthal example whose results are identical to those of the LWD problems shown in Section 4 (this can be done by employing a simple re-scaling technique, see $[27])$.

\section{Conclusions}

Non-fitting grids are easier to generate than fitting grids, and thus lead to simpler and possibly more efficient implementations. For the particular case of 3D Maxwell's equations with a constant magnetic permeability, we know from [7] that the optimal convergence of the magnetic field is preserved for first-order edge finite elements.

In this work, we evaluate the impact of non-fitting grids on the accuracy of the simulated resistivity logs in the context of borehole logging applications. To this end, we consider 2.5D Maxwell's equations that we discretize using high-order edge and nodal elements (for the tangential and normal components of the magnetic field with respect to the Fourier plane, respectively). We present four numerical experiments, which are representative of LWD and deep-azimuthal applications. 

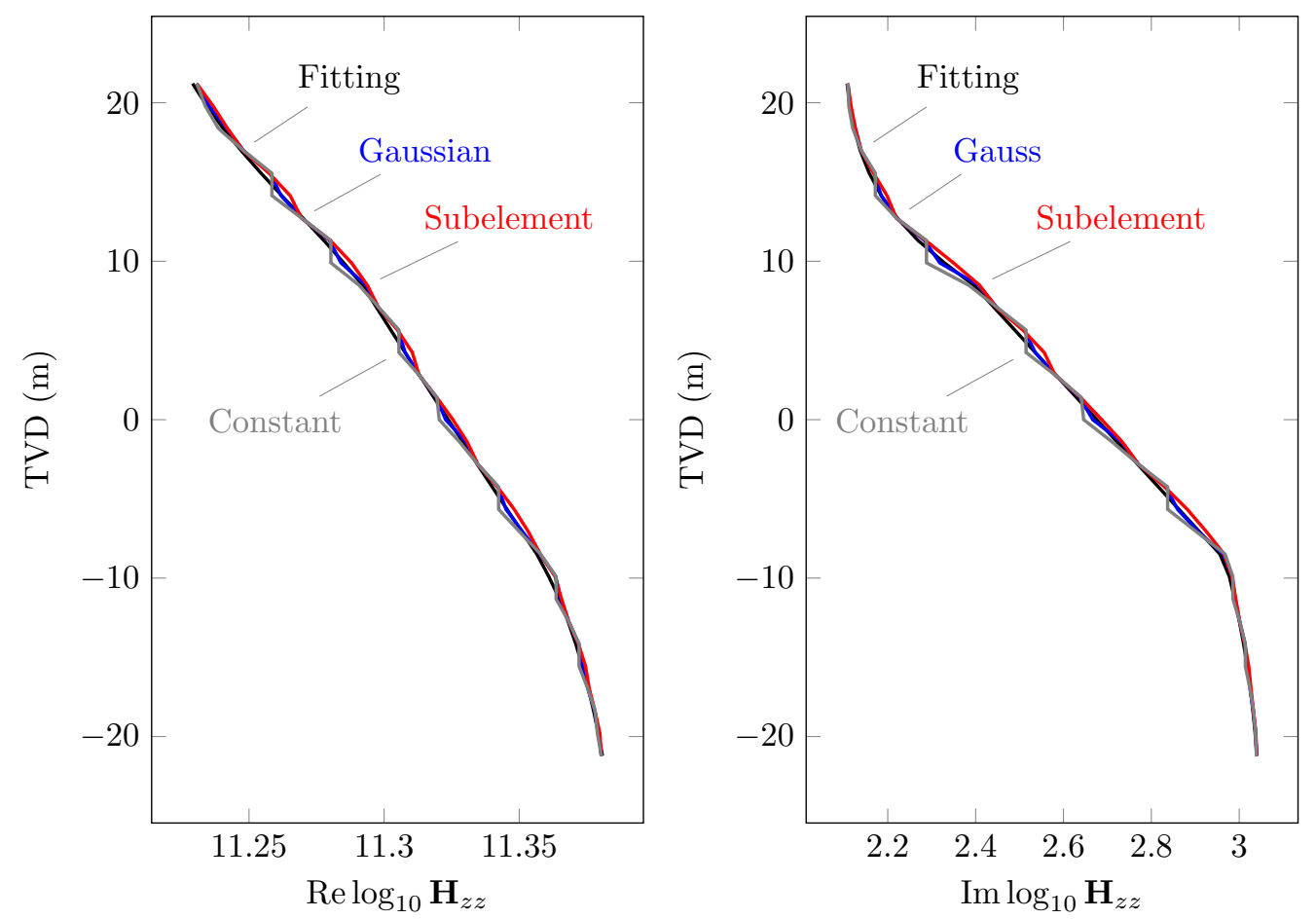

FigURE 14. $\mathbf{H}_{z z}$ component for the deep-azimuthal logging experiment
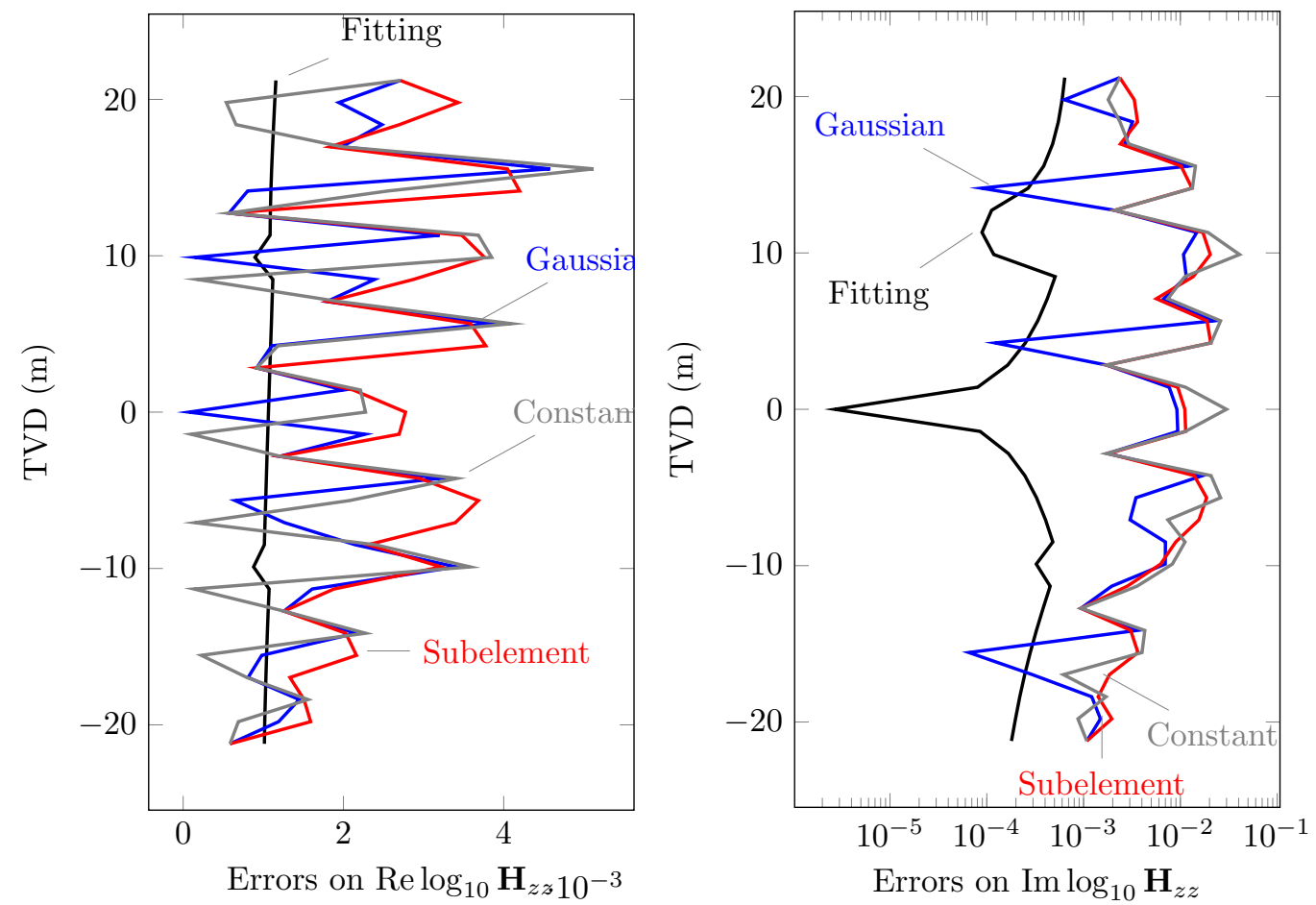

FigURE 15. $\mathbf{H}_{z z}$ component for the deep-azimuthal logging experiment 
FE SIMULATIONS OF RESISTIVITY MEASUREMENTS USING NON-FITTING GRIDS
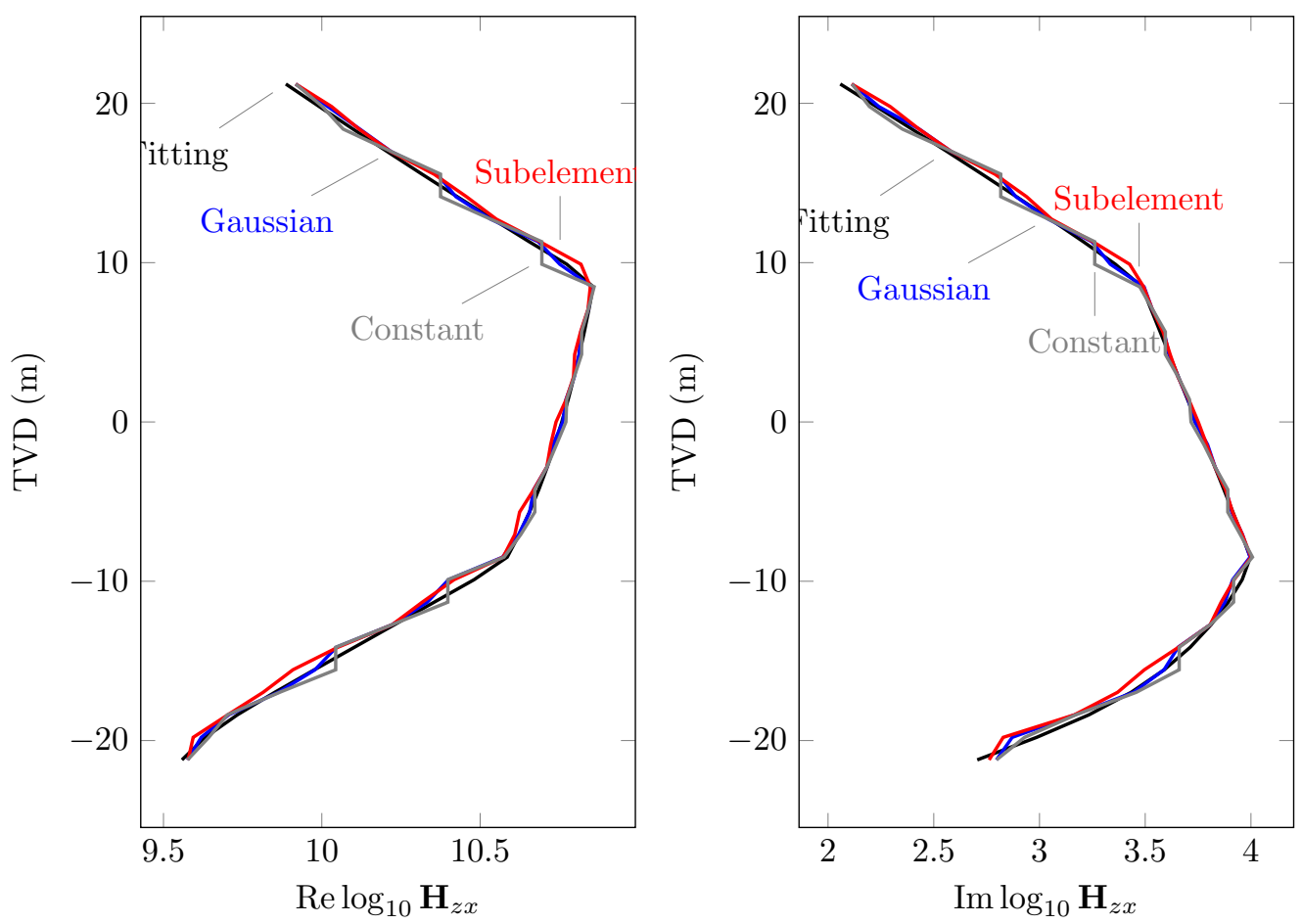

FIGURE 16. $\mathbf{H}_{z x}$ component for the deep-azimuthal logging experiment
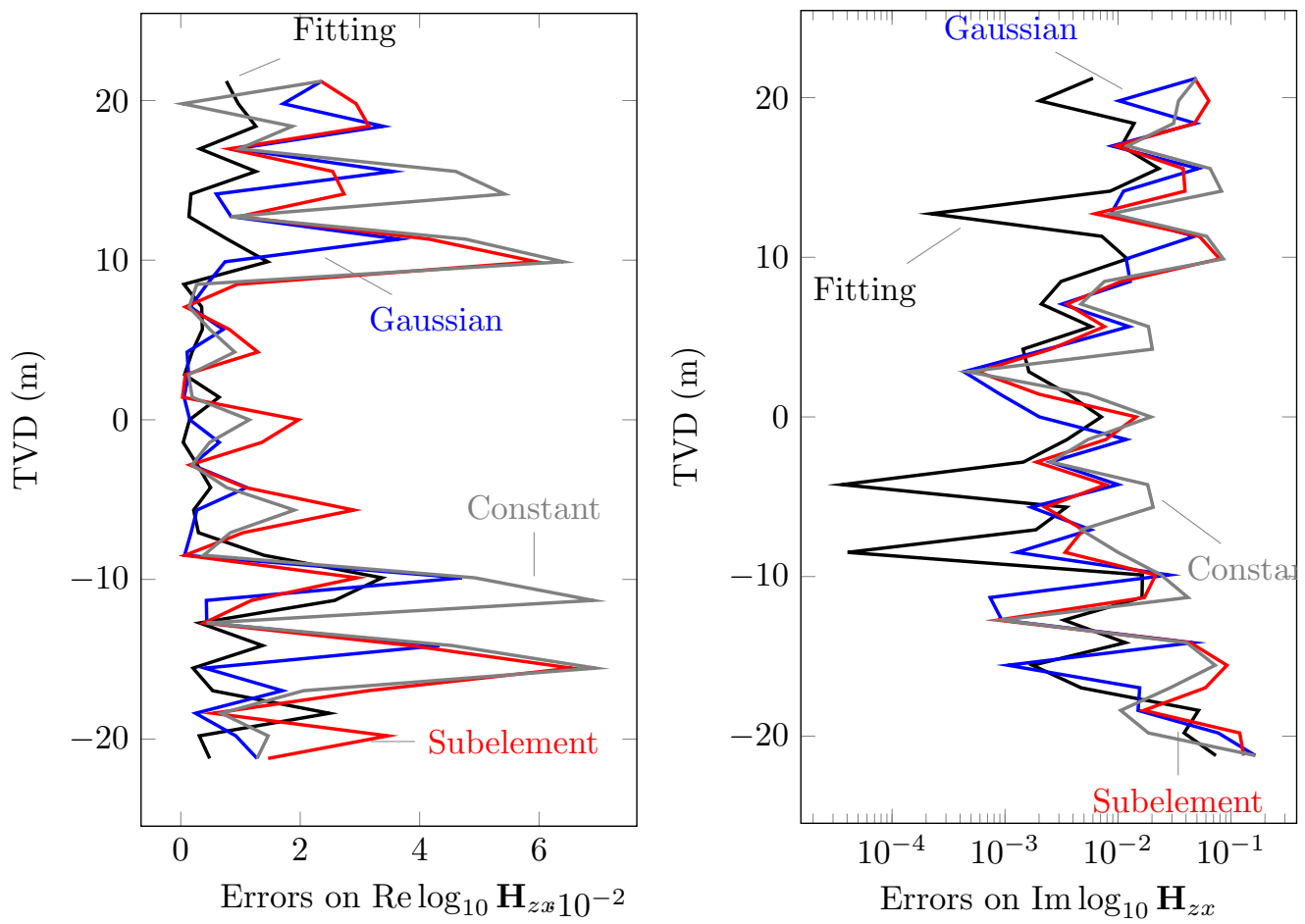

FIGURE 17. $\mathbf{H}_{z x}$ component for the deep-azimuthal logging experiment 
In all considered examples, the use of fitting and non-fitting grids provide approximations of similar quality, provided that an appropriate quadrature scheme is employed for non-fitting grids. We compare three different strategies that we call "Constant parameter", "Gaussian" and "Subelement".

Both Gaussian and Subelement integration techniques provide accurate solutions, while the fastest Constant integration technique often (but not always) lead to inaccurate results. The Gaussian integration technique seems more convenient for quadrangular elements, as non-affine mappings are easily handled. On the other hand, the Subelement integration technique may be more suitable for straight triangular elements.

\section{ACKNowledgements}

The authors have received funding from the European Union's Horizon 2020 research and innovation programme under the Marie Sklodowska-Curie grant agreement No 644602, the Projects of the Spanish Ministry of Economy and Competitiveness with reference MTM2016-76329-R (AEI/FEDER, EU), and MTM2016-81697-ERC/AEI, the BCAM "Severo Ochoa" accreditation of excellence SEV-2013-0323, and the Basque Government through the BERC 2014-2017 program, and the Consolidated Research Group Grant IT649-13 on "Mathematical Modeling, Simulation, and Industrial Applications (M2SI)".

\section{REFERENCES}

1. A. Abubakar, T.M. Habashy, V.L. Druskin, L. Knizhnerman, and D. Alumbaugh, 2.5d forward and inverse modeling for interpreting low-frequency electromagnetic measurements, Geophysics 73 (2008), no. 4, F165F177.

2. A. Abubakar, P.M. van de Berg, and T.M. Habashy, An integral equation approeach for 2.5-dimensional forward and inverse electromagnetic scattering, Geophys. J. Int. 165 (2006), 744-762.

3. I. Babuška, G. Caloz, and J.E. Osborn, Special finite element methods for a class of second order elliptic problems with rough coefficients, SIAM J. Numer. Anal. 31 (1994), no. 4, 945-981.

4. H. Barucq, T. Chaumont-Frelet, and C. Gout, Stability analysis of heterogeneous Helmholtz problems and finite element solution based on propagation media approximation, Mathematics of Computation 86 (2016), no. 307, 2129-2157.

5. M. Bittar, d R. Beste J. Klein a, G. Hu, M. Wu, J. Pitcher, C. Golta, G. Althoff, M. Sitka, V. Minosyan, and M. Paulk, A new azimuthal deep-reading resistivity tool for geosteering and advanced formation evaluation, SPE Reservoir Evaluation \& Engineering (2009), 270-279.

6. A.C. Cangellaris and D.B. Wright, Analyses of the numerial error caused by the stair-stepped approximation of a conducting boundary in FDTD simulations of electromagnetic phenomena, IEEE transactions on antennas and propagation 39 (1991), no. 10, 1518-1525.

7. T. Chaumont-Frelet, S. Nicaise, and D. Pardo, Finite element approximation of electromagnetic fields using non-fitting meshes for geophysics, submitted to SIAM J. Numer. Anal. (2017).

8. P.G. Ciarlet, The finite element method for elliptic problems, SIAM, 1978.

9. S. Davydycheva, V. Druskin, and T. Habashy, An efficient finite-difference scheme for electromagnetic logging in $3 d$ anisotropic inhomogeneous media, Geophysics 68 (2003), no. 5, 1525-1536.

10. S. Davydycheva, D. Homan, and G. Minerbo, Triaxial induction tool with electrode sleeve: FD modeling in $3 D$ geometries, Journal of Applied Geophysics 67 (2009), 98-108.

11. D. Garcia, D. Pardo, L. Dalcin, M. Paszyński, N. Collier, and V.M. Calo, The value of continuity: Refined isogeometric analysis and fast direct solvers, Computer Methods in Applied Mechanics and Engineering 213216 (2017), 353-361.

12. T.M. Habashy and A. Abubakar, A generalized material averaging formulation for modelling the electromagnetic fields, J. of Electromagn. Waves and Appl. 21 (2007), no. 9, 1145-1159.

13. A.Q. Howard and W.C. Chew, Electromagnetic borehole fields in a layered, dipping-bed environment with invasion, Geophysics 57 (1992), no. 3, 451-465.

14. G. Kristensson, Homogenization of the Maxwell equations in an anisotropic material, Radio Science 38 (2003), no. 2, VIC 19-1-VIC-19-8.

15. R.J. Leveque and Z. Li, The immersed interface method for elliptic equations with discontinuous coefficients and singular sources, SIAM J. Number. Anal. 31 (1994), no. 4, 1019-1044.

16. H. Liu, Principles and applications of well logging, 1 ed., Springer Mineralogy, Springer-Verlag Berlin Heidelberg, 2017.

17. P. Monk, Finite element methods for Maxwell's equations, Oxford University Press, 2003.

18. S. Moskow, V. Druskin, T. Habashy, P. Lee, and S. Davydycheva, A finite difference scheme for elliptic equations with rough coefficients using a cartesian grid nonconforming to interfaces, SIAM J. Numer. Anal. 36 (1999), no. 2, 442-464. 
19. I. Muga, D. Pardo, P.J. Matuszyk, and C. Torres-Verdín, Semi-analytical response of acoustic logging measurements in frequency domain, Computer and Mathematics with Applications 70 (2015), 314-329.

20. M.J. Nam, D. Pardo, C. Torres-Verdín, S. Hwang, K.G. Park, and C. Lee, Simulation of eccentricity effects on shot- and long-normal logging measurements using a fourier-hp-finite-element method, Exploration Geophysics 41 (2010), 118-127.

21. J.C. Nédélec, Mixed finite elements in $R^{3}$, Numer. Math. 35 (1980), 315-341.

22. R.E. Nimmer, J.L. Osiensky, and A.M. Binley, Effects of borehole fill on resistivity investigations, Symposium on the Application of Geophysics to Engineering and Environmental Problems 2003 (2003), 116-130.

23. D. Pardo, L. Demkowicz, C. Torres-Verdín, and M. Paszynski, Two-dimensional high-accuracy simulation of resistivity logging-while-drilling (lwd) measurements using a self-adaptive goal-oriented hp finite element method, SIAM J. Appl. Math. 66 (2006), no. 6, 2085-2106.

24. D. Pardo, M.J. Nam, C. Torres-Verdín, M.G. Hoversten, and L. Garay, Simulation of marine controlled source electromagnetic measurements using a parallel fourier hp-finite element method, Comput Geosci 67 (2011), $15-23$.

25. D. Pardo, M. Paszyński, N. Collier, J. Alvarez, L. Dalcin, and V.M. Calo, The value of continuity: Refined isogeometric analysis and fast direct solvers, SeMA Journal 57 (2012), 107-134.

26. D. Pardo and C. Torres-Verdín, Fast 1d inversion of logging-while-drilling resistivity measurements for improved estimation of formation resistivity in high-angle and horizontal wells, Geophysics 80 (2015), no. 2, E111-E124.

27. A. Rodríguez-Rozaz, D. Pardo, and C. Torres-Verdín, Advances toward the fast simulation of 2.5d lwd and deep azimuthal resistivity tools, Proceedings of Joint Industry Research Consortium of Formation Evaluation (Sixteenth Annual Meetin), Austin, TX, USA. (2016), 1-4.

28. J.M. Salazar and C. Torres-Verdín, Quantitative comparison of processes of oil- and water-based mud-filtrate invasion and corresponding effects on borehole resistivity measurements, Geophysics 74 (2009), no. 1, E57-E73.

29. D.R. Smith and J.B. Pendry, Homogenization of metamaterials by field averaging (invited paper), J. Opt. Soc. Am. B 23 (2006), no. 3, 391-403.

30. R. Tilsley-Baker, A. Hartmann, M. Sviridov, O. Sanabria, J. Skillings, M. Kjolleberg, J.E. Barbosa, L. Loures, P. Swalf Pearson, and B. Morani, The Importance of Extra-Deep Azimuthal Resistivity in Reservoir Development: An Update on Recent Field Experiences, (2015).

31. M.J. Wilt, D.L. Alumbaugh, H.F. Morrison, A. Becker, K.H. Lee, and M. Deszcz-Pan, Crosswell electromagnetic tomography: System design considerations and field results, Geophysics 60 (1995), no. 3, 871-885. 